Physics

\title{
Work and heat probability distributions in out-of-equilibrium systems
}

\author{
Alberto Imparato \\ Dipartimento di Fisica, INFN-Sezione di Torino, CNISM-Sezione di Torino, Politecnico di Torino, C.so Duca degli Abruzzi \\ 24, 10129 Torino, Italy \\ Luca Peliti \\ Dipartimento di Scienze Fisiche, INFN-Sezione di Napoli, CNISM-Sezione di Napoli, Università "Federico II", Complesso \\ Monte S. Angelo, 80126 Napoli, Italy
}

November 24, 2006

\begin{abstract}
We review and discuss the equations governing the distribution of work done on a system which is driven out of equilibrium by external manipulation, as well as those governing the entropy flow to a reservoir in a nonequilibrium system. We take advantage of these equations to investigate the path phase transition in a manipulated meanfield Ising model and the large-deviation function for the heat flow in the asymmetric exclusion process with periodically varying transition probabilities. To cite this article: A. Imparato, L. Peliti, C. R. Physique 6 (2005).

\section{Résumé}

Distributions du travail et de la chaleur dans des systèmes hors équilibre. Nous passons en revue et discutons les équations régissant la distribution du travail effectué sur un système manipulé hors d'équilibre, ainsi que celles qui régissent le flux d'entropie vers un reservoir dans un système hors d'équilibre. Nous exploitons ces équations dans l'étude de la transition de phase dans les chemins d'un modèle d'Ising champ moyen manipulé et de la fonction des grandes déviations pour le flux d'entropie dans le modèle d'exclusion asymétrique à probabilités de transition périodiques dans le temps. Pour citer cet article: A. Imparato, L. Peliti, C. R. Physique 6 (2005).

Key words: Nonequilibrium processes ; Work distribution ; Entropy flow

Mots-clés : Procès hors équilibre; Distribution du travail ; Flux d'entropie
\end{abstract}

\section{Introduction}

In the recent years we have seen an important outburst of activity in the field of nonequilibrium thermodynamics and statistical mechanics, sparked by the discovery of a number of results of remarkable generality and

Email addresses: alberto.imparato@polito.it (Alberto Imparato), peliti@na.infn.it (Luca Peliti). 
impact $[1,2,3,4,5,6,7,8,9,10,11,12,13,14,15,16,17,18]$. Some of these results yield predictions on the properties of the distribution of the work performed on a system as it is manipulated, while others describe properties of the distribution of the entropy generated in the nonequilibrium process. It is clear that in order to make good use of this information, it is advisable to investigate in detail the properties of the distribution of both work and heat in a manipulated system.

In the present Contribution we discuss the work and the heat distribution in some systems, pointing out some aspects that we have found interesting enough to be brought to the attention of our colleagues. We first consider the expression of the generating function for the work distribution in a "large" system. Although our results formally hold in the limit of infinite size, they are of interest also for systems small enough that the relevant energy barriers are of the order of a few $k_{\mathrm{B}} T$, well within the range in which work fluctuations can be observed. In a large system, as already pointed out by Ritort [19] and discussed in [20], the generating function of the work distribution is dominated by the contribution of phase space paths which satisfy an ordinary differential equation akin to a classical equation of motion. We have found that in some situations these paths can exhibit a singularity, for some protocols, as a function of the variable conjugate to the accumulated work. This singularity is similar to a phase transition taking place in the space of paths. We shall exhibit a simple model in which this phenomenon takes place and attempt to define the corresponding phase diagram.

We also consider the heat flow distribution in a general Markov process, whose differential equations were derived by Lebowitz and Spohn [8]. We were able to evaluate the solution of this equation for the asymmetric exclusion model with periodically varying transition probabilities, and to exhibit that the Gallavotti-Cohen [5] symmetry also holds in this case.

\section{The generating function for the work distribution}

We first briefly review the derivation of the generating function for the work distribution of manipulated systems, which may be found in [20]. Let us consider a system with discrete states $i$ of energy $H_{i}(\mu)$, where $\mu$ is a parameter which is manipulated according to some protocol $\mu(t)$, starting at $t=0$. The evolution of the system is described by a markovian stochastic process: given, for any two states $i, j$, the transition rate $k_{i j}(t)$ from state $j$ to state $i$ at time $t$, the system satisfies the set of differential equations

$$
\frac{\partial p_{i}}{\partial t}=\sum_{j(\neq i)}\left[k_{i j}(t) p_{j}(t)-k_{j i}(t) p_{i}(t)\right]
$$

where $p_{i}(t)$ is the probability that the system is found at state $i$ at time $t$. Let $p_{i}^{\text {eq }}(\mu)$ represent the canonical equilibrium distribution corresponding to a given value of $\mu$. We have

$$
p_{i}^{\mathrm{eq}}(\mu)=\frac{\mathrm{e}^{-\beta H_{i}(\mu)}}{Z_{\mu}}
$$

where $Z_{\mu}=\sum_{i} \mathrm{e}^{-\beta H_{i}(\mu)}=\mathrm{e}^{-\beta F_{\mu}}$ is the partition function corresponding to the value $\mu$ of the parameter, and $F_{\mu}$ the relative free energy. The transition rates $k_{i j}(t)$ are compatible with the equilibrium distribution $p_{i}^{\text {eq }}(\mu)$, i.e., one has, for all $i$,

$$
\sum_{j(\neq i)}\left[k_{i j}(t) p_{j}^{\mathrm{eq}}(\mu(t))-k_{j i}(t) p_{i}^{\mathrm{eq}}(\mu(t))\right]=0
$$

We assume that the system is at equilibrium at $t=0$, and therefore, that $p_{i}(t)$ satisfies the initial condition

$$
p_{i}(t=0)=p_{i}^{\mathrm{eq}}(\mu(0))
$$

We now consider the joint probability distribution $\Phi_{i}(W, t)$ that the system is found in state $i$, having received a work $W$, at time $t$. The set of differential equations satisfied by the distribution functions $\Phi_{i}(W, t) \operatorname{reads}$

$$
\frac{\partial \Phi_{i}}{\partial t}=\sum_{j(\neq i)}\left[k_{i j}(t) \Phi_{j}(W, t)-k_{j i}(t) \Phi_{i}(W, t)\right]-\dot{\mu} H_{i}^{\prime}(\mu(t)) \frac{\partial \Phi_{i}}{\partial W} .
$$


The joint probability distribution $\Phi_{i}(W, t)$ satisfies the initial condition $\Phi_{i}(W, 0)=\delta(W) p_{i}^{\text {eq }}(\mu(0))$. Then the state-independent work probability distribution $P(W, t)$ is defined by $P(W, t)=\sum_{i} \Phi_{i}(W, t)$. It is convenient to introduce the generating function of $\Phi_{i}$ with respect to the work distribution, defined by

$$
\Psi_{i}(\lambda, t)=\int \mathrm{d} W \mathrm{e}^{\lambda W} \Phi_{i}(W, t)
$$

We assume that $\Phi_{i}(W, t)$ vanishes fast enough, as $|W| \rightarrow \infty$, for $\Psi_{i}(\lambda, t)$ to exist for any $\lambda$. The function $\Psi_{i}$ satisfies, for all $\lambda$, the initial condition $\Psi_{i}\left(\lambda, t_{0}\right)=p_{i}^{\mathrm{eq}}(\mu(0))$, and evolves according to the differential equation

$$
\partial_{t} \Psi_{i}(\lambda, t)=\sum_{j(\neq i)}\left[k_{i j} \Psi_{j}-k_{j i} \Psi_{i}\right]+\lambda \dot{\mu} \frac{\partial H_{i}(\mu(t))}{\partial \mu} \Psi_{i}(\lambda, t) .
$$

Exploiting (3), it is easy to verify that, if $\lambda=-\beta$, the solution of (7) with its initial condition reads

$$
\Psi_{i}(-\beta, t)=\frac{\mathrm{e}^{-\beta H_{i}(\mu(t))}}{Z_{\mu(0)}}=\frac{Z_{\mu(t)}}{Z_{\mu(0)}} p_{i}^{\mathrm{eq}}(\mu(t))
$$

We can thus straightforwardly check that the solution of (7) verifies the Jarzynski equality [6]:

$$
\left\langle\mathrm{e}^{-\beta W}\right\rangle=\frac{Z_{\mu(t)}}{Z_{\mu(0)}}=\mathrm{e}^{-\beta(F(\mu(t))-F(\mu(0)))} .
$$

It is thus possible, in principle, to evaluate the probability distribution function of the work $W$ by solving the equations (5) or (7) for all the microscopic states $i$. This approach has been implemented in [21] for a simple model of a biopolymer.

The approach discussed in the previous section becomes quickly unwieldy as the complexity of the system increases: the dimension of the system (5) is equal to the number of microscopic states of the system. When the system considered is characterized by a large number of degrees of freedom, it is convenient to introduce some collective variables, and an effective free energy, in order to reduce the complexity of the problem. The assumption underlying this approach is that the system reaches on a comparatively short time scale a quasiequilibrium state constrained by the instantaneous value of the collective coordinate. Thus, on the the time scale of the experiment, the state of the system can be well summarized by the collective coordinate, with the corresponding free energy playing the role of the hamiltonian.

Let us now consider a system characterized by a generic equilibrium free energy function $\mathcal{F}_{\mu}(M)$, where $\mu$ is again the parameter which is manipulated, and $M$ is some collective (mean-field) variable. We assume that the system dynamics is stochastic and markovian: let $P(M, t)$ denote the probability distribution function of the variable $M$ at time $t$, then its time evolution is described by the differential equation

$$
\frac{\partial P}{\partial t}=\widehat{\mathcal{H}} P
$$

where $\widehat{\mathcal{H}}$ is a differential operator which depends on the parameter $\mu$. We require that the operator $\widehat{\mathcal{H}}$ is compatible with the equilibrium distribution function of the system, i.e., that the relation

$$
\widehat{\mathcal{H}} \mathrm{e}^{-\beta \mathcal{F}_{\mu}(M)}=0
$$

holds for any value of $\mu$.

The work done on a system during the manipulation, along a given stochastic trajectory $M(t)$, is given by

$$
W=\int_{0}^{t} \mathrm{~d} t^{\prime} \dot{\mu}\left(t^{\prime}\right) \frac{\partial \mathcal{F}_{\mu}\left(M\left(t^{\prime}\right)\right)}{\partial \mu} .
$$

Using the same arguments as for the discrete case, one finds that the time evolution of the joint probability distribution $\Phi(M, W, t)$ of $M$ and $W$ is described by the differential equation

$$
\frac{\partial \Phi}{\partial t}=\widehat{\mathcal{H}} \Phi-\dot{\mu} \frac{\partial \mathcal{F}_{\mu}}{\partial \mu} \frac{\partial \Phi}{\partial W}
$$

It can be easily shown that the solution of (13) identically satisfies the Jarzynski equality [22]. 
Equation (13) becomes much easier to treat if one introduces the generating function $\Psi(M, \lambda, t)$ for the work distribution:

$$
\Psi(M, \lambda, t)=\int \mathrm{d} W \mathrm{e}^{\lambda W} \Phi(M, W, t)
$$

Equation (13) thus becomes

$$
\frac{\partial \Psi}{\partial t}=\widehat{\mathcal{H}} \Psi+\lambda \dot{\mu} \frac{\partial \mathcal{F}_{\mu}}{\partial \mu} \Psi
$$

with the initial condition $\Psi(M, \lambda, 0)=p_{\mu(0)}^{\mathrm{eq}}(M)=\mathrm{e}^{-\beta \mathcal{F}_{\mu(0)}(M)} / Z_{\mu(0)}$.

As shown in [22], one can derive a path integral representation of the solution of (15), taking for the differential operator $\widehat{\mathcal{H}}$ the expression

$$
\widehat{\mathcal{H}} \cdot=\sum_{k=0}^{\infty} \frac{\partial^{k}}{\partial M^{k}}\left\{g_{k}(M) \cdot\right\} .
$$

(The coefficients $g_{k}(M)$ also depend on $\mu$, but this dependence is understood to lighten the notation.) One obtains the formal solution

$$
\Psi\left(M, \lambda, t_{\mathrm{f}}\right)=\int \mathrm{d} M_{0} \int_{M(0)=M_{0}}^{M\left(t_{\mathrm{f}}\right)=M} \mathcal{D} \gamma \mathcal{D} M \exp \{\mathcal{S}[\gamma, M]\} \Psi\left(M_{0}, \lambda, 0\right),
$$

where $\mathcal{S}[\gamma, M]=\int_{0}^{t_{\mathrm{f}}} \mathrm{d} t \mathcal{L}(t)$. The "lagrangian" $\mathcal{L}$ is given by

$$
\mathcal{L}(t)=\left.\left(\gamma \dot{M}+\mathcal{H}(\gamma, M)+\lambda \dot{\mu} \frac{\partial \mathcal{F}_{\mu}}{\partial \mu}\right)\right|_{\gamma(t), M(t), \mu(t)},
$$

where the "hamiltonian" $\mathcal{H}(\gamma, M)$ is defined by

$$
\mathcal{H}(\gamma, M)=\sum_{k=0}^{\infty} \gamma^{k} g_{k}(M)
$$

Let $N$ indicate the size of the system, and let us define the "intensive quantity" $m=M / N$. We can thus define, in the thermodynamic limit $N \rightarrow \infty, m=$ const., the densities

$$
f_{\mu}(m)=\lim _{N \rightarrow \infty} \frac{\mathcal{F}_{\mu}(N m)}{N}, \quad H(\gamma, m)=\lim _{N \rightarrow \infty} \frac{\mathcal{H}(\gamma, N m)}{N}, \quad \ell(t)=\lim _{N \rightarrow \infty} \frac{\mathcal{L}(t)}{N},
$$

with $\ell(t)=\gamma \dot{m}+H(\gamma, m)+\lambda \dot{\mu} \partial f_{\mu} / \partial \mu$. As discussed in $[19,22]$, when the system size $N$ is large enough, the path integral in (17) is dominated by the classical path $\left(\gamma_{\mathrm{c}}(t), m_{\mathrm{c}}(t)\right)$, solution of the equations

$$
\frac{\delta \mathcal{S}}{\delta \gamma(t)}=0 \Longrightarrow \dot{m}=-\frac{\partial H}{\partial \gamma} ; \quad \frac{\delta \mathcal{S}}{\delta m(t)}=0 \Longrightarrow \dot{\gamma}=\frac{\partial H}{\partial m}+\lambda \dot{\mu} \frac{\partial^{2} f_{\mu}}{\partial m \partial \mu}
$$

We shall now see that by requiring the system is in equilibrium before the manipulation starts, we impose an initial condition on these equations. In order to evaluate the integral over $M_{0}$ in (17) with the saddle-point method, we note that $\Psi(M, \lambda, 0)$ appearing on its rhs, is given by $p_{\mu(0)}^{\text {eq }}(M)$. Furthermore, from the definition of $\ell(t),(20)$, it follows that

$$
\int_{0}^{t_{\mathrm{f}}} \mathrm{d} t \ell(t)=m_{t_{\mathrm{f}}} \gamma_{t_{\mathrm{f}}}-m_{0} \gamma_{0}+\int_{0}^{t_{\mathrm{f}}} \mathrm{d} t\left[-\dot{\gamma} m+H+\lambda \dot{\mu} \partial_{\mu} f_{\mu}\right] .
$$

Thus, substituting (22) into (17), and taking the derivative with respect to $m_{0}=M_{0} / N$, we obtain the saddle-point condition

$$
\gamma(t=0)=-\left.\beta \frac{\partial f_{\mu}}{\partial m}\right|_{t=0} .
$$

In this way one can devise a strategy to evaluate $\Psi\left(M, \lambda, t_{\mathrm{f}}\right)$ for a given manipulation protocol $\mu(t)$, when the system size $N$ is large enough. One has to solve the classical evolution equations (21) with a two-point boundary 
condition: namely, (23) should be imposed at $t=0$, and the condition $N m\left(t_{\mathrm{f}}\right)=M$ should be imposed at the final time $t_{\mathrm{f}}$. Once the relevant classical path $\left(\gamma_{\mathrm{c}}(t), m_{\mathrm{c}}(t)\right)$ has been evaluated, one can obtain the action density $s\left[\gamma_{\mathrm{c}}, m_{\mathrm{c}}\right]=\lim _{N \rightarrow \infty} \mathcal{S}\left[\gamma_{\mathrm{c}}, N m_{\mathrm{c}}\right] / N$ from the expression $s\left[\gamma_{\mathrm{c}}, m_{\mathrm{c}}\right]=\int_{0}^{t_{\mathrm{f}}} \mathrm{d} t \ell(t)$. We are interested in the state-independent work probability distribution

$$
P\left(W, t_{\mathrm{f}}\right)=\int \mathrm{d} \lambda \mathrm{e}^{-\lambda W} \Gamma\left(\lambda, t_{\mathrm{f}}\right),
$$

where we have defined $\Gamma\left(\lambda, t_{\mathrm{f}}\right)=\int \mathrm{d} M \Psi\left(M, \lambda, t_{\mathrm{f}}\right)$. We shall now see that evaluating $\Gamma\left(\lambda, t_{\mathrm{f}}\right)$ identifies a welldefined boundary condition on $\gamma_{\mathrm{c}}\left(t_{\mathrm{f}}\right)$. We have indeed

$$
\Gamma\left(\lambda, t_{\mathrm{f}}\right)=\int \mathrm{d} M \mathrm{~d} M_{0} \int_{M(0)=M_{0}}^{M\left(t_{\mathrm{f}}\right)=M} \mathcal{D} \gamma \mathcal{D} M \exp \left[N \int \mathrm{d} t \ell(t)\right] \Psi\left(M_{0}, \lambda, 0\right) .
$$

In order to evaluate the integral over $M$ with the saddle point method, we notice that, upon derivation of the rhs of (22) with respect to $m_{t_{\mathrm{f}}}$, we obtain the condition $\gamma_{\mathrm{f}} \equiv \gamma\left(t_{\mathrm{f}}\right)=0$. Thus, the equation of motions (21) have to be solved with the initial and the final conditions that we have derived: let $\left(\gamma_{\mathrm{c}}^{*}(t), m_{\mathrm{c}}^{*}(t)\right)$ denote the solution of equations (21) satisfying these conditions. For each value of $\lambda$, taking into account its initial condition, we obtain the following saddle point estimation for $\Gamma\left(\lambda, t_{\mathrm{f}}\right)$ :

$$
\Gamma\left(\lambda, t_{\mathrm{f}}\right) \propto \frac{\exp [N g(\lambda)]}{Z_{0}}
$$

where

$$
g(\lambda)=\int_{0}^{t_{\mathrm{f}}} \mathrm{d} t \ell_{\mathrm{c}}^{*}(t)-\beta f_{\mu_{0}}\left(m_{0}^{*}\right) .
$$

In this equation, $\ell_{\mathrm{c}}^{*}(t)$ is $\ell(t)$ evaluated along the classical path $\left(\gamma_{\mathrm{c}}^{*}(t), m_{\mathrm{c}}^{*}(t)\right)$. In order to evaluate the integral on the rhs of (24), we use the saddle point method again, and obtain

$$
P\left(N w, t_{\mathrm{f}}\right)=\mathcal{N} \exp \left\{N\left[-\lambda^{*}(w) w+g\left(\lambda^{*}(w)\right)\right]\right\}, \quad \text { with } \quad g^{\prime}\left(\lambda^{*}\right)=w,
$$

where $\mathcal{N}$ is a normalization constant. Notice that the saddle point estimate for $P\left(W, t_{\mathrm{f}}\right)$ obtained in this way, implies that the distribution becomes more and more sharply peaked around its maximum value as $N \rightarrow \infty$. This is compatible with the expectation that the work fluctuations becomes relatively smaller as the size of the system increases, and in the limit $N \rightarrow \infty$, which can be thought as the limit of a macroscopic system, no work fluctuations are observed, and the work done on the system during the manipulation takes one single value, corresponding to the most probable value of $P\left(W, t_{\mathrm{f}}\right)$.

\section{A path phase transition}

In this section we consider an Ising model in mean-field approximation, which evolves according to a FokkerPlanck equation, whose differential operator reads

$$
\widehat{\mathcal{H}} \cdot=\omega_{0} N \frac{\partial}{\partial M}\left[\left(\frac{\partial \mathcal{F}}{\partial M}\right) \cdot+\beta^{-1} \frac{\partial}{\partial M} \cdot\right],
$$

leading to the hamiltonian

$$
H(\gamma, m)=\omega_{0}\left[\gamma\left(\frac{\partial f}{\partial m}\right)+\beta^{-1} \gamma^{2}\right]
$$

where the free energy density $f(m)=\mathcal{F}(N m) / N$ is given by

$$
f(m)=-\frac{J}{2} m^{2}-h m+\beta^{-1}\left[\left(\frac{1+m}{2}\right) \log \left(\frac{1+m}{2}\right)+\left(\frac{1-m}{2}\right) \log \left(\frac{1-m}{2}\right)\right] .
$$


We take the magnetic field $h(t)$ as the external parameter that varies with the time and drives the system out of equilibrium. The equations of motion (21) thus become

$$
\dot{m}=-\frac{\partial H}{\partial \gamma}=-\omega_{0} \frac{\partial f}{\partial m}-2 k_{\mathrm{B}} T \omega_{0} \gamma ; \quad \dot{\gamma}=\frac{\partial H}{\partial m}+\lambda \dot{\mu} \frac{\partial^{2} f}{\partial m \partial \mu}=\omega_{0} \frac{\partial^{2} f}{\partial m^{2}} \gamma-\lambda \dot{h},
$$

The magnetic field $h(t)$ is taken to vary according to the linear protocol

$$
h(t)=h_{0}+\left(h_{1}-h_{0}\right) \frac{t}{t_{\mathrm{f}}} ; \quad 0 \leq t \leq t_{\mathrm{f}}
$$

Here we consider the case of Ising model below the transition temperature, i.e., $J=1.1$, and the initial and final value of the magnetic field $h(t)$ are taken to be $h_{0}=-h_{1}=-1$. In the present section we set $\beta=1 / k_{\mathrm{B}} T=1$. We plot in figure 1 the probability distribution of the work done on the model, as obtained from (28), for two values of the manipulation rate. In the same figure the histograms of the work obtained by simulating the process are plotted. The process is simulated by integrating the corresponding Langevin equation, using the Heun algorithm [23,24]. The agreement with the curves as obtained from (28) is satisfactory. In the insets of the same figure, we plot the quantity $\hat{P}(w)$ defined by

$$
\hat{P}(w)=\exp [-\beta N w] P(w)
$$

On the one hand we find $\int \mathrm{d} w \hat{P}(w)=\exp [-\beta \Delta F]=1$ as predicted by the JE (9), while on the other hand the histogram obtained by the simulations exhibits no point (no realization of the process) with $w<0=\Delta F$. Thus the work distribution obtained by the simulation of the process cannot reliably be used for estimating $\Delta F$. This is a typical example of how the lack of knowledge of the tails of the work distributions in micro-manipulations experiments hinders the possibility of using (9) to evaluate free energy differences.
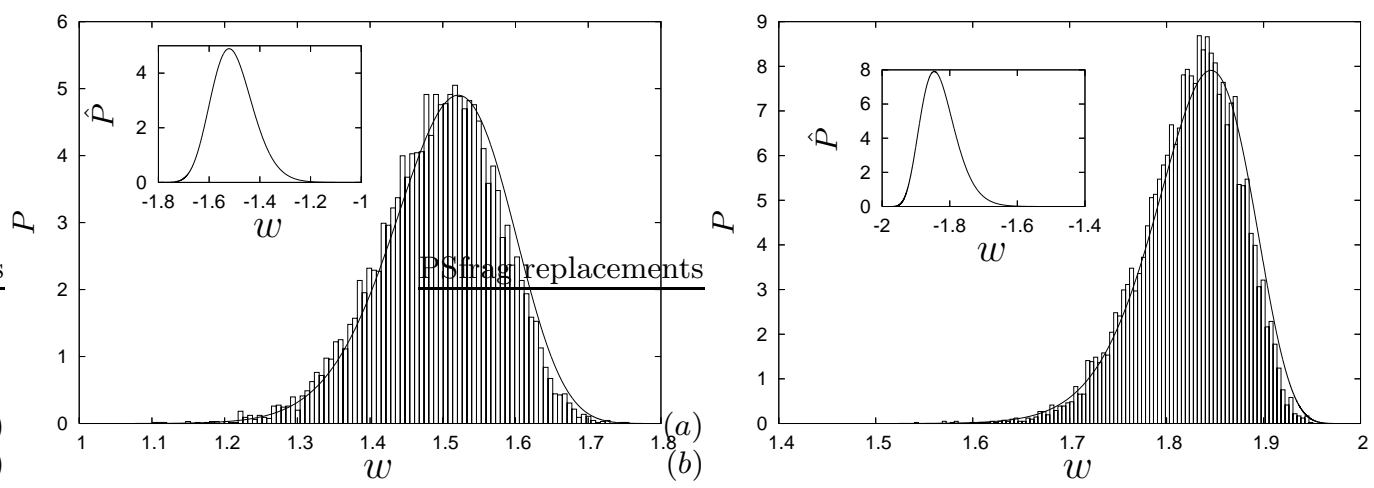

Figure 1. Probability distribution function $P(w)$ for the system described by the differential operator (29) with equilibrium free energy (31), manipulated according to the protocol (33), with $J_{1}=1.1, h_{0}=-h_{1}=-1$, and $t_{\mathrm{f}}=2$ (left panel), $t_{\mathrm{f}}=4$ (right panel). Continuous line: probability density $P(w)$ of the work "per spin" $w=W / N$, with $N=100$. The histogram of the work is obtained by 10000 simulations of the process, see text. Insets: $\hat{P}(w)$ as given by (34), whose integral verifies the Jarzynski equality.

We now consider the classical paths $m_{\mathrm{c}}^{*}(t, \lambda)$, solutions of equations (32), and thus contributing to the probability distribution $P(w)$ via (28). In the left panel of figure 2, we plot $m_{\mathrm{c}}^{*}$ as a function of $t$ for different values of $\lambda$, obtained by numerical solution of equations $(32)$, for $t_{\mathrm{f}}=2$ : we observe that the trajectory $m_{\mathrm{c}}^{*}(t, \lambda)$ varies continuosly as $\lambda$ is varied. As a consequence, since the work done on the system along each trajectory $m_{\mathrm{c}}^{*}(t, \lambda)$ reads $w=-\int_{0}^{t_{\mathrm{f}}} \mathrm{d} t^{\prime} \dot{h}\left(t^{\prime}\right) m_{\mathrm{c}}^{*}\left(t^{\prime}, \lambda\right), w$ turns out to be a continuous function of $\lambda$ (figure 2, right panel). Furthermore, since $w$ and the saddle point value $\lambda^{*}$ are related by (28), the function $g(\lambda)$ as given by (27) is a differentiable function with respect to $\lambda$, as shown in the inset of figure 2 , right panel. We now consider a faster protocol, $t_{\mathrm{f}}=0.2$ : the results are plotted in figure 3 . One can clearly see that the classical paths $m_{\mathrm{c}}^{*}(t, \lambda)$ exhibit a discontinuity for $\lambda=0.5$, jumping from negative to positive values. Accordingly, $w\left(\lambda^{*}\right)$ exhibits a discontinuity at $\lambda^{*}=0.5$, as shown in the right panel of figure 3. This is reflected in the appearence of a cusp in the function $g(\lambda)$, at $\lambda=1 / 2$, see the inset of figure 3. We find that for any value of $r=\left(h_{1}-h_{0}\right) / t_{\mathrm{f}}$ it is always possible to find a value of $J=J^{*}(r)$ such that for $J>J^{*}(r)$ the function $w\left(\lambda^{*}\right)$ exibits a discontinuity at $\lambda=1 / 2$, and thus the trajectories $m_{\mathrm{c}}^{*}(t, \lambda)$ exhibit a path phase separation. In figure 4 , left panel, we show the system phase diagram: in the upper 

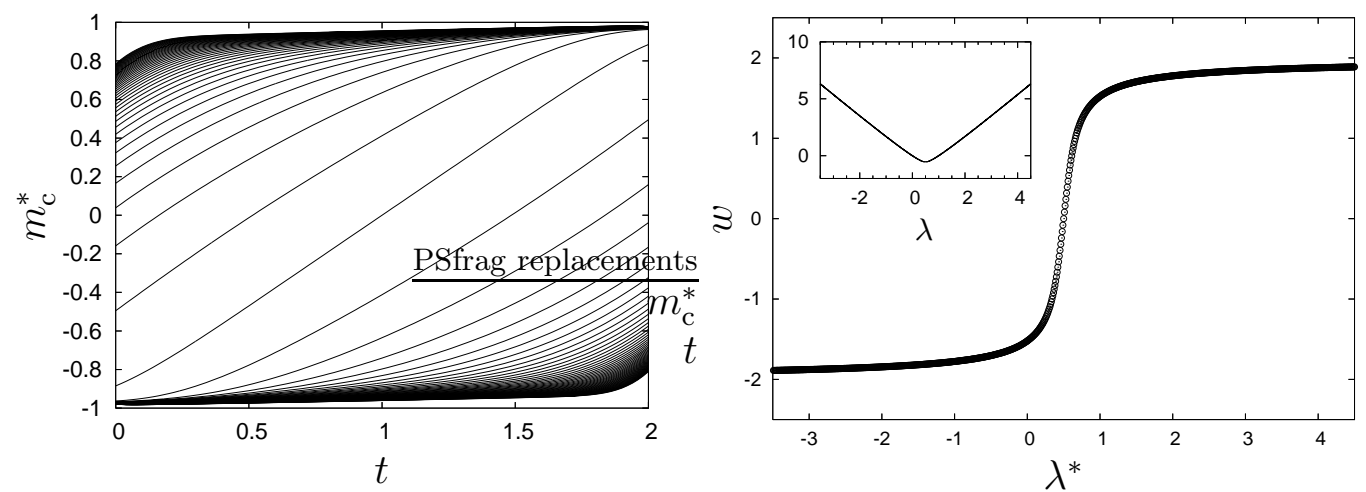

Figure 2. Left: plot of $m_{\mathrm{c}}^{*}$ as a function of $t$ for different values of $\lambda$, with $J=1.1, h_{0}=-h_{1}=-1$, and $t_{\mathrm{f}}=2$. The values of $\lambda$ vary between $\lambda=-5$ (bottom curve) and $\lambda=5$ (top curve), with a step $\Delta \lambda=0.2$. Right: plot of $w$ as a function of $\lambda^{*}$, as defined by (28). Inset: plot of $g$ as a function of $\lambda$ as given by (27).
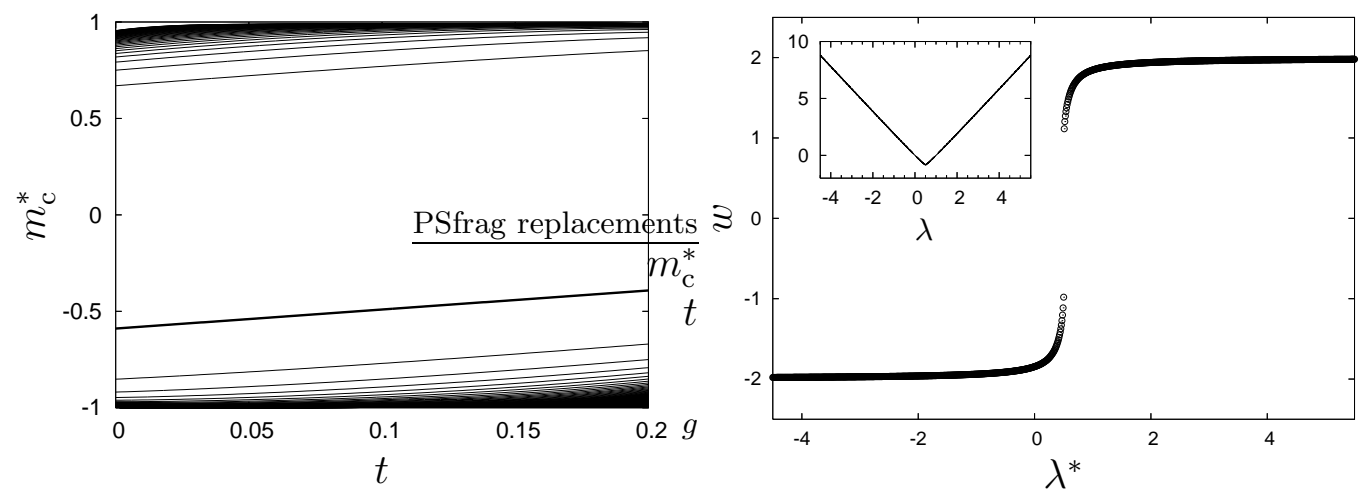

Figure 3. Left: Plot of $m_{\mathrm{c}}^{*}$ as a function of $t$ for different values of $\lambda$, with $J=1.1, h_{0}=-h_{1}=-1$, and $t_{\mathrm{f}}=0.2$. The values of $\lambda$ vary between $\lambda=-5$ (bottom curve) and $\lambda=5$ (top curve), with a step $\Delta \lambda=0.2$. Thick line: $\lambda=0.5$. Right: plot of $w$ as a function of $\lambda^{*}$, as defined by (28). Inset: plot of $g$ as a function of $\lambda$ as defined by (27).
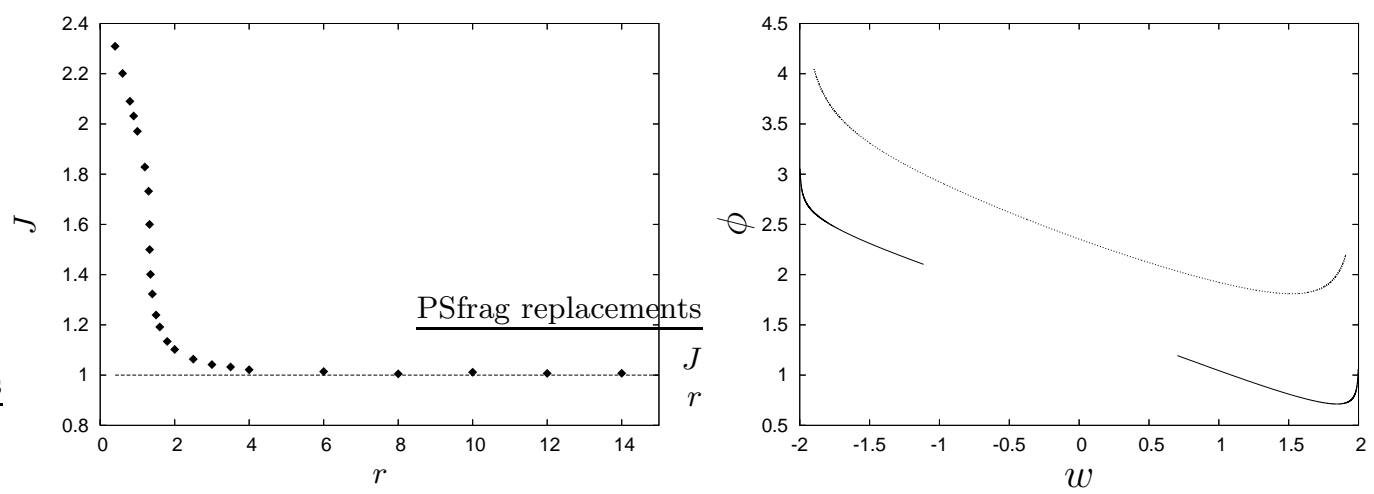

Figure 4. Left: Ising model phase diagram, in the $r=\left(h_{1}-h_{0}\right) / t_{\mathrm{f}}, J$ plane. In the upper right part of the diagram the system exhibits path phase separation, while in the lower left part no phase separation is found. The points correspond to the value $J^{*}(r)$ such that for $J>J^{*}(r)$ the function $w\left(\lambda^{*}\right)$ exibits a discontinuity at $\lambda=1 / 2$. Right: Path Helmholtz free energy for the system described by the differential operator (29), manipulated according to the protocol (33). Full line: $J=1.1, h_{0}=-h_{1}=-1$, and $t_{\mathrm{f}}=0.2$. Dotted line: $J=1.1, h_{0}=-h_{1}=-1$, and $t_{\mathrm{f}}=2$. The curves are arbitrarily shifted for clarity.

right part of the diagram the system exhibits path phase separation, while in the lower left part the trajectories $m_{\mathrm{c}}^{*}(t, \lambda)$ vary continuously as $\lambda$ is varied.

Let us now define the function 


$$
\phi(w)=-\lim _{N \rightarrow \infty} \frac{1}{N} \log P\left(N w, t_{\mathrm{f}}\right)
$$

The functions $g(\lambda)$ and $\phi(w)$ are related by a Legendre transformation:

$$
\phi(w)=\inf _{\lambda}(-g(\lambda)+\lambda w)=-g\left(\lambda^{*}(w)\right)+\lambda^{*}(w) w
$$

where $\lambda^{*}(w)$ is the solution of (28). Thus $\lambda$ and $w$ act like thermodynamically conjugate variables. These functions can be thus interpreted in terms of path thermodynamics: $g(\lambda)$ can be viewed as a path Gibbs free energy, while $\phi(w)$ is the corresponding Helmholtz free energy. This analogy was first pointed out in $[19,20]$.

How do we interpret the singularity in $g(\lambda)$ ? In strict analogy with thermodynamics, the discontinuity of $g^{\prime}(\lambda)$ with respect to its independent variable $\lambda$ corresponds to the linear behavior of its Legendre transform $\phi(w)$ between $\left(w_{+}, \phi\left(w_{+}\right)\right)$and $\left(w_{-}, \phi\left(w_{-}\right)\right)$, where $w_{ \pm}$are the values of $w$ either side of the discontinuity. Thus the "path phase coexistence" appears as an exponential dependence of $P\left(N w, t_{\mathrm{f}}\right)=\exp (-N \phi(w))$ on $W$ in a certain interval. The presence of such exponential tails was conjectured in [19] for a system of independent spins, but we were only able to exhibit them in an interacting system like the present one.

\section{The distribution of heat flow in a Markov process}

In this section we discuss the equation governing the time evolution of the probability distribution function of the entropy which flows into the enviroment surrounding a stochastic system which evolves across its phase space. We assume that the system at issue has a discrete phase space and its time evolution is a stochastic markovian process described by (1). For simplicity, we consider a stochastic dynamics with a discrete small time scale $\tau$, such that the jumps between states take place at discrete times $t_{k}=k \tau$. We consider a generic path $\omega$ defined by $\omega(t)=i_{k}$ iff $t_{k} \leq t<t_{k+1}$, with $k=0,1, \ldots, M$, with $t_{M+1}=t_{\mathrm{f}}$, and define the time-reversed path $\widetilde{\omega}$ by $\widetilde{\omega}(t)=i_{k}$ for $\tilde{t}_{k+1} \leq t<\tilde{t}_{k}$, where $\tilde{t}=t_{0}+t_{\mathrm{f}}-t$. Let us define the quantity $Q(\omega)$ by

$$
Q(\omega)=-\ln \left[\frac{\mathcal{P}(\omega)}{\widetilde{\mathcal{P}}(\widetilde{\omega})}\right]=-\sum_{k=1}^{M} \ln \left[\frac{K_{i_{k} i_{k-1}}\left(t_{k}\right)}{K_{i_{k-1} i_{k}}\left(t_{k}\right)}\right]
$$

where $\mathcal{P}(\omega)$ is the probability of the forward path $\omega$ (conditioned by its initial state $i_{0}$ ) and $\widetilde{\mathcal{P}}(\widetilde{\omega})$ is the probability of the time-reversed path $\widetilde{\omega}$, conditioned by its initial state $i_{M} \equiv i_{\mathrm{f}}$ and subject to the time-reversed protocol $\widetilde{K}_{i j}(t)=K_{i j}(\tilde{t})[8,9,10,16]$. We have assumed that, if $K_{i j}(t)>0$ at any time $t$, one also has $K_{j i}(t)>0$.

It is worth noting that, if the detailed balance conditions holds for the transition rates $K_{i j}(t)$, and the energy $H_{i}(t)$ is associated to the state $i$ of the system, we have $K_{j i}(t) / K_{i j}(t)=\exp \left\{\left[H_{i}(t)-H_{j}(t)\right] / T\right\}$, and thus $T \ln \left[K_{j i}(t) / K_{i j}(t)\right]$ represents the heat exchanged with the reservoir in the jump from state $j$ to state $i$. (In this section we set $k_{\mathrm{B}}=1$.) Thus the quantity $Q(\omega)$, defined by (37), is the entropy which flows into the reservoir as the system evolves along the path $\omega[8,14,15,25]$. Let us define $\Delta s_{i j}$ as the entropy which flows into the reservoir as a result of the jump of the system from state $j$ to state $i \Delta s_{i j}=\log \left[K_{j i}(t) / K_{i j}(t)\right]$. The differential equation governing the time evolution of the joint probability distribution function $\Phi_{i}(Q, t)$ reads $[25]$

$$
\frac{\partial \Phi_{i}(Q, t)}{\partial t}=\sum_{j(\neq i)}\left\{K_{i j}\left[\sum_{n=0}^{\infty} \frac{\left(-\Delta s_{i j}\right)^{n}}{n !} \frac{\partial^{n} \Phi_{j}(Q, t)}{\partial Q^{n}}\right]-K_{j i} \Phi_{i}(Q, t)\right\}
$$

By introducing, for each $i$, the generating function $\Psi_{i}(\lambda, t)=\int \mathrm{d} Q \exp (\lambda Q) \Phi_{i}(Q, t)$, and taking into account the expression of $\Delta s_{i j}$, we obtain the master equation

$$
\frac{\partial \Psi_{i}(\lambda, t)}{\partial t}=\sum_{j(\neq i)}\left[K_{i j}\left(\frac{K_{j i}}{K_{i j}}\right)^{\lambda} \Psi_{j}(\lambda, t)-K_{j i} \Psi_{i}(\lambda, t)\right]=\sum_{j} H_{i j}(\lambda) \Psi_{j}(\lambda, t) .
$$

which was first derived by Lebowitz and Spohn in [8]. In the case of time-independent transition rates $K_{i j}$, or of transition rates which depend periodically on the time, it can be useful, in order to evaluate the distribution function $\Phi(Q, t)=\sum_{i} \Phi(Q, t)$, to introduce the large-deviation function. In the long-time limit, the generating 
function $\Psi(\lambda, t)=\sum_{i} \Psi_{i}(\lambda, t)$ is dominated by the maximum eigenvalue $g(\lambda)$ of the matrix $\mathrm{H}(\lambda)=\left(H_{i j}(\lambda)\right)$, which appears in the master equation (39). Therefore, we have, for long times $t$,

$$
\Psi(\lambda, t) \propto \exp [\operatorname{tg}(\lambda)] .
$$

By using the last equation and using the definition of the generating function, one obtains the probability distribution of the entropy flow in the long time limit:

$$
\Phi(Q, t)=\int \frac{\mathrm{d} \lambda}{2 \pi \mathrm{i}} \mathrm{e}^{-\lambda Q} \Psi(\lambda, t) \propto \mathrm{e}^{t g\left(\lambda^{*}\right)-\lambda^{*} Q},
$$

where $\lambda^{*}$ is the saddle point value implicitly defined by $\partial g /\left.\partial \lambda\right|_{\lambda^{*}}=Q / t$. If we introduce the entropy flow per unit time $q=Q / t$, we obtain the large-deviation function

$$
f(q) \equiv g\left(\lambda^{*}\right)-\lambda^{*} q=\lim _{t \rightarrow \infty} \frac{1}{t} \log \Phi(t q, t) .
$$

Note that the functions $g(\lambda)$ and $f(q)$ are Legendre transform of each other, and can be then interpreted in terms of path thermodynamics: $g(\lambda)$ can be viewed as a path Gibbs free energy, while $f(q)$ is the corresponding Helmholtz free energy. The connection between the generating function $\Psi(\lambda)$ and the thermodynamic formalism for dynamical systems [26] has been investigated in Ref. [27].

If the system is characterized by a small number of states, one can explicitly solve the equations (39), and thus obtain the total generating function $\Psi(\lambda, t) \equiv \sum_{i} \Psi_{i}(\lambda, t)=\left\langle\mathrm{e}^{\lambda Q}\right\rangle$. While on the one hand this direct approach becomes rapidly impracticable, as the system phase space size increases, on the other hand (39) suggests a practical computational scheme to evaluate the generating function $\Psi(\lambda, t)$. Since $\Psi(\lambda, t)=\int \mathcal{D} \omega_{t} \mathcal{P}\left(\omega_{t}\right) \mathrm{e}^{\lambda Q\left(\omega_{t}\right)}$, we have

$$
\frac{\partial \Psi(\lambda, t)}{\partial \lambda}=\langle Q\rangle_{\lambda} \Psi(\lambda, t)
$$

where $\langle\ldots\rangle_{\lambda}$ is the average in the weighted ensemble $\mathcal{P}\left(\omega_{t}\right) \exp \left[\lambda Q\left(\omega_{t}\right)\right] / \Psi(\lambda, t)$, where $\Psi(\lambda, t)=Z_{\lambda}$ is the "partition function" of this ensemble, which will be called the " $\lambda$-ensemble" in the following. The solution of (43) thus reads

$$
\Psi(\lambda, t)=\exp \left[\int_{0}^{\lambda} \mathrm{d} \lambda^{\prime}\langle Q\rangle_{\lambda^{\prime}}\right]
$$

Following [25], in the present paper we consider a procedure to evaluate $\Psi(\lambda, t)$ which generates trajectories in a suitable entropy-flow weighted ensemble. The direct simulation of trajectories in the $\lambda$-ensemble is hindered by the fact that one should already know the exact expression of the ensemble partition function, i.e., the function $\Psi(\lambda, t)$, which is the unknown quantity at issue.

To avoid the problem of the direct evaluation of $\Psi(\lambda, t)$, following [28], we introduce a generic functional of the paths $\Pi(\omega)$, and write

$$
\langle Q\rangle_{\lambda}=\frac{\int \mathcal{D} \omega_{t}\left(Q\left(\omega_{t}\right) / \Pi\left(\omega_{t}\right)\right) \Pi\left(\omega_{t}\right) \mathcal{P}\left(\omega_{t}\right) \mathrm{e}^{\lambda Q\left(\omega_{t}\right)}}{\int \mathcal{D} \omega_{t}\left(1 / \Pi\left(\omega_{t}\right)\right) \Pi\left(\omega_{t}\right) \mathcal{P}\left(\omega_{t}\right) \mathrm{e}^{\lambda Q\left(\omega_{t}\right)}}=\frac{\langle Q / \Pi\rangle_{\lambda, \Pi}}{\langle 1 / \Pi\rangle_{\lambda, \Pi}},
$$

where $\langle\ldots\rangle_{\lambda, \Pi}$ indicates the average in the new $\mathcal{P}\left(\omega_{t}\right) \Pi\left(\omega_{t}\right) \exp \left[\lambda Q\left(\omega_{t}\right)\right]$ ensemble, which will be indicated as the $(\lambda, \Pi)$-ensemble in the following.

We choose the functional of the path $\Pi(\omega)$ as discussed in [25]. The probability of a given path $\omega$ reads

$$
\mathcal{P}(\omega)=\Omega_{i_{N}, i_{N-1}}\left(t_{N-1}\right) \Omega_{i_{N-1}, i_{N-2}}\left(t_{N-2}\right) \ldots \Omega_{i_{1}, i_{0}}\left(t_{0}\right) p_{i_{0}}^{0}
$$

where the transition probabilities $\Omega_{i, j}(t)$ are defined as $\Omega_{i, j}=\tau K_{i_{j}}(t)$, and $\Omega_{i, i}=1-\sum_{j(\neq i)} \Omega_{j i}(t)$. We now define the new transition probabilities $\widetilde{\Omega}_{i, j}=\tau K_{i j}\left(K_{j i} / K_{i j}\right)^{\lambda}$, and $\widetilde{\Omega}_{i, i}=1-\sum_{j(\neq i)} \widetilde{\Omega}_{j i}$, and choose the functional $\Pi(\omega)$, such that $[25]$

$$
\Pi(\omega)=\prod_{k=1}^{M} \Pi_{i_{k}, i_{k-1}}\left(t_{k}\right), \quad \text { with } \quad \Pi_{i j}(t)= \begin{cases}1, & \text { if } i \neq j \\ \widetilde{\Omega}_{j j}(t) / \Omega_{j j}(t), & \text { if } i=j .\end{cases}
$$


Recalling the definition of $Q(\omega),(37)$, we obtain that the probability in the $(\lambda, \Pi)$-ensemble is given by

$$
\mathcal{P}(\omega) \Pi(\omega) \exp [\lambda Q(\omega)]=\widetilde{\Omega}_{i_{N}, i_{N-1}} \widetilde{\Omega}_{i_{N}, i_{N-1}} \ldots \widetilde{\Omega}_{i_{1}, i_{0}} p_{i_{0}}^{0}
$$

and thus $\langle Q\rangle_{\lambda}$ can be evaluated by using (45), for the particular choice of $\Pi$, as given by (47). Note that (48) implies that the one can generate a trajectory in the $(\lambda, \Pi)$-ensemble by simply simulating the process with the $\widetilde{\Omega}_{i, j}(t)$ transition probabilities.

In [25], the the feasibility of the method was illustred by applying it to a nonequilibrium system characterized by a large phase space, and evolving according to a stochastic dynamics, namely the simple asymmetric exclusion process (ASEP) [29]. Such a system consists in a one-dimensional lattice gas on a lattice of $L$ sites. Each site of the model is either empty or occupied by at most one particle. Each particle can jump into an empty nearest neighbor site with transition rates per time unit $K_{+}$(rightward) and $K_{-}$(leftward). The system is kept in an out-ofequilibrium steady state since its first and last site are in contact with two particle reservoirs, at densities $\rho_{A}$ and $\rho_{B}$ respectively. By taking $\rho_{A}>\rho_{B}$ and $K_{+}>K_{-}$, one observes a net particle current from the left to the right reservoir. In [25] we considered an ASEP model with constant parameters $\rho_{A}, \rho_{B}, K_{+}, K_{-}$. Here we consider a system with time-dependent parameters. We take $K_{+}=1, K_{-}=K_{-}^{0}(1+\sin (2 \pi t))+\epsilon$, with $K_{-}^{0}=0.75, \epsilon=10^{-3}$, $\alpha=1, \gamma=0.27, \beta=1, \delta=0.27$, where $\alpha-\gamma$ are the rates of jump from-into the left $A$ reservoir, respectively, and $\beta-\delta$ are the rates of jump into-from the right $B$ reservoir, respectively. With this choice of parameters, at $t=2 k \pi$, we have $\rho_{A}=0.75, \rho_{B}=0.25$ [30]. The duration of a single trajectory is taken to be $t_{\mathrm{f}}=10 \cdot 2 \pi$. A
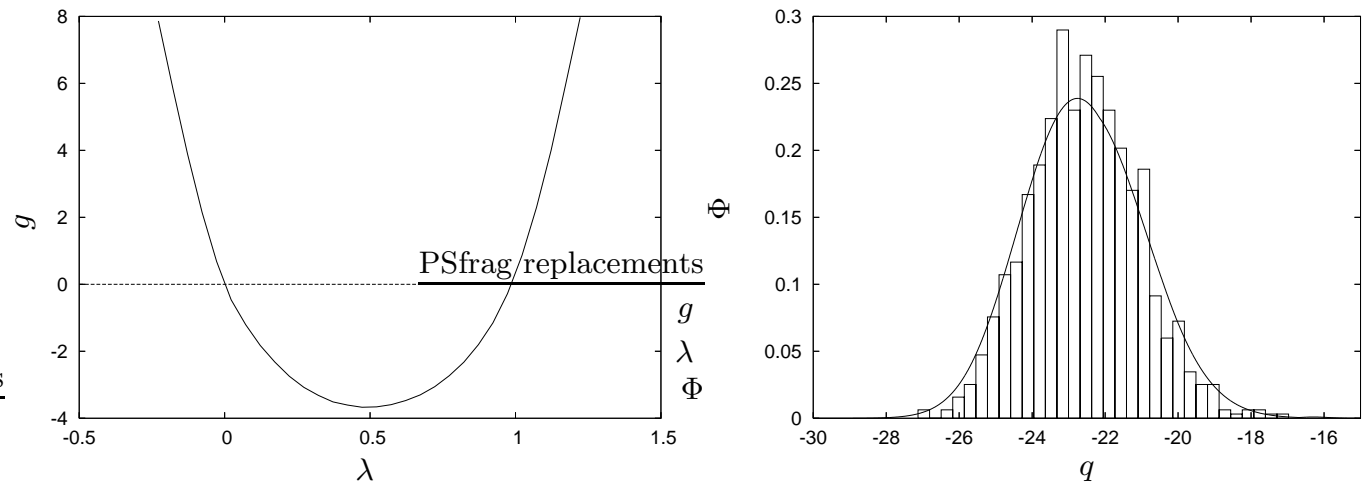

Figure 5. Left: Plot of $g(\lambda)$ as obtained by combining (40) and (44), for the ASEP model. The function $g(\lambda)$ vanishes for $\lambda=0,1$ which corresponds to the normalization condition and to Seifert's fluctuation relation [16] respectively. Right: Histogram of the entropy flow per time unit $q$, corresponding to 1000 unbiased trajectories. Full line: probability distribution function $\Phi\left(t_{\mathrm{f}} q, t_{\mathrm{f}}\right)$, obtained with the trajectory sampling algorithm.

direct evaluation of the function $g(\lambda)$ by solving the $2^{100}$ equations (39) is out of question. We thus apply our trajectory simulation approach to the ASEP model. We consider trajectories with elementary time step $\tau=0.01$ : at each time the transition probability between two states is given by the transition matrix $\widetilde{K}_{i j}(t)$. For each value of $\lambda$ we generate $\mathcal{N}=10000$ sample trajectories and calculate the entropy flow $Q$, as defined by (37), for each trajectory. Then, for the given value of $\lambda$, by averaging over the $\mathcal{N}$ trajectories, we compute the quantity $\langle Q\rangle_{\lambda}$ using (45). Finally, by combining (40) and (44), we obtain the function $g(\lambda)$ which governs the long time behavior of $\Psi(\lambda, t)$. This function is plotted in figure 5, left panel. It can be seen that it vanishes at $\lambda=0,1$ and is symmetric with respect to $\lambda=1 / 2$. The fact that $g(0)=0$ corresponds trivially to the normalization condition over all the possible trajectories. On the other hand, the fact that the function $g$ vanishes at $\lambda=1$, is a non trivial result, and corresponds to Seifert's fluctuation theorem [16]. The symmetry around $\lambda=1 / 2$ corresponds to the GallavottiCohen fluctuation relation. We are now able to calculate the large-deviation function $f(q)$ defined in (42). We check as follows that the quantity $f(q)$ actually gives the entropy distribution function $\Phi(q, t) \propto \exp [t f(q)]$ for the present model. We simulate the unbiased diffusion process by using the transition matrix $K_{i j}(t)$, and measure the entropy flow along 1000 trajectories. We then plot the histogram of the measured entropy flow per time unit, together with the function $\exp [t f(q)]$, see figure 5 , right panel. The agreement between the histogram and the predicted entropy distribution $\Phi(q, t)$ is excellent. 


\section{Discussion}

We have seen that the equations governing the evolution of the work and heat flow distributions in out-ofequilibrium systems can be harnassed to yield interesting information. We have analyzed the generating function of the work distribution in "large" manipulated systems and shown that it may exhibit a "path phase transition", which corresponds to the presence of an exponential behavior in some work interval. On the other hand, we have shown that it is possible to evaluate the large deviation function for the entropy flow in a stochastic process via a biased simulation technique, and applied the method to exhibit the Gallavotti-Cohen symmetry in an out-ofequilibrium system with periodically varying parameters. A similar result has been recently obtained by Ge and collaborators [31].

Acknowledgements We are grateful to Pierre Gaspard and Christian van den Broeck for having given us the opportunity of taking part in this exciting meeting. We also thank C. Jarzynski and U. Seifert for their interest in our work.

\section{References}

[1] G. N. Bochkov, Y. E. Kuzovlev, Nonlinear fluctuation-dissipation relations and stochastic models in nonequilibrium thermodynamics i. generalized fluctuation-dissipation theorem, Physica 106 (3) (1981) 443-479.

[2] G. N. Bochkov, Y. E. Kuzovlev, Nonlinear fluctuation-dissipation relations and stochastic models in nonequilibrium thermodynamics: Ii. kinetic potential and variational principles for nonlinear irreversible processes, Physica 106 (3) (1981) $480-520$.

[3] D. J. Evans, E. G. D. Cohen, G. P. Morriss, Probability of second law violations in shearing steady states, Phys. Rev. Lett. $71(15)(1993) 2401-2404$

[4] D. J. Evans, D. J. Searles, Steady states, invariant measures, and response theory, Phys. Rev. E 52 (6) (1995) $5839-5848$.

[5] G. Gallavotti, E. G. D. Cohen, Dynamical ensembles in stationary states, J. Stat. Phys. 80 (5) (1995) 931-970.

[6] C. Jarzynski, Nonequilibrium equality for free energy differences, Phys. Rev. Lett. 78 (14) (1997) 2690-2693.

[7] J. Kurchan, Fluctuation theorem for stochastic dynamics, J. Phys. A: Math. Gen. 31 (1998) 3719-3729.

[8] J. L. Lebowitz, H. Spohn, A Gallavotti-Cohen-type symmetry in the large deviation functional for stochastic dynamics, J. Stat. Phys. 95 (1) (1999) 333-365.

[9] G. E. Crooks, Entropy production fluctuation theorem and the nonequilibrium work relation for free energy differences, Phys. Rev. E 60 (3) (1999) 2721-2726.

[10] G. E. Crooks, Path-ensemble averages in systems driven far from equilibrium, Phys. Rev. E 61 (3) (2000) $2361-2366$.

[11] G. Hummer, A. Szabo, Free energy reconstruction from nonequilibrium single-molecule pulling experiments, PNAS 98 (7) (2001) 3658-3661.

[12] T. Hatano, S. Sasa, Steady state thermodynamics of langevin systems, Physical Review Letters 86 (2001) 3463.

[13] C. Maes, On the origin and the use of fluctuation relations for the entropy, Sém. Poincaré 2 (2003) $29-62$.

[14] P. Gaspard, Time-reversed dynamical entropy and irreversibility in Markovian random processes, J. Stat. Phys. 117 (2004) 599-615.

[15] P. Gaspard, Brownian motion, dynamical randomness and irreversibility, New J. of Physics 7 (2005) 77.

[16] U. Seifert, Entropy production along a stochastic trajectory and an integral fluctuation theorem, Phys. Rev. Lett. 95 (4) (2005) 040602 .

[17] A. Imparato, L. Peliti, Fluctuation relations for a driven brownian particle, Phys. Rev. E 74 (2) (2006) 026106.

[18] B. Cleuren, C. V. den Broeck, R. Kawai, Fluctuation and dissipation of work in a joule experiment, Physical Review Letters 96 (2006) 050601.

[19] F. Ritort, Work and heat fluctuations in two-state systems, J. of Statistical Mechanics (Theory and Experiment) (2004) P10016.

[20] A. Imparato, L. Peliti, Work-probability distribution in systems driven out of equilibrium, Phys. Rev. E 72 (4) (2005) 046114. 
[21] A. Imparato, L. Peliti, Work probability distribution in single-molecule experiments, Europhys. Lett. 69 (2005) $643-650$.

[22] A. Imparato, L. Peliti, Work distribution and path integrals in general mean-field systems, Europhys. Lett. 70 (2005) $740-746$.

[23] A. Greiner, W. Strittmatter, J. Hohnerkamp, Numerical integration of stochastic differential equations, J. Stat. Phys. 51 (1988) 95-108.

[24] R. Mannella, Integration of stochastic differential equations on a computer, Int. J. of Modern Physics C 13 (2002) $1177-1195$.

[25] A. Imparato, L. Peliti, The distribution function of entropy flow in stochastic systems, J. of Statistical Mechanics (Theory and Experiment) (2007) L02001.

[26] D. Ruelle, Thermodynamic formalism, 2nd Edition, Cambridge University Press, Cambridge, 2004.

[27] V. Lecomte, C. Appert-Rolland, F. van Wijland, Chaotic properties of systems with markov dynamics, Phys. Rev. Lett. 95 (2005) 010601.

[28] H. Oberhofer, C. Dellago, P. L. Geissler, Biased sampling of nonequilibrium trajectories: Can fast switching simulations outperform conventional free energy calculation methods?, J. Phys. Chem. B 109 (2005) 6902-6915.

[29] G. M. Schütz, E. Domany, Phase transitions in an exactly soluble one-dimensional exclusion process, J. Stat. Phys. 72 (1993) $277-296$.

[30] C. Enaud, B. Derrida, Large deviation functional of the weakly asymmetric exclusion process, J. Stat. Phys. 114 (2004) 537-562.

[31] H. Ge, D. Q. Jiang, M. Qian, A simple discrete model of Brownian motors: Time-periodic Markov chains, J. Stat. Phys. 123 (2006) 831-859. 


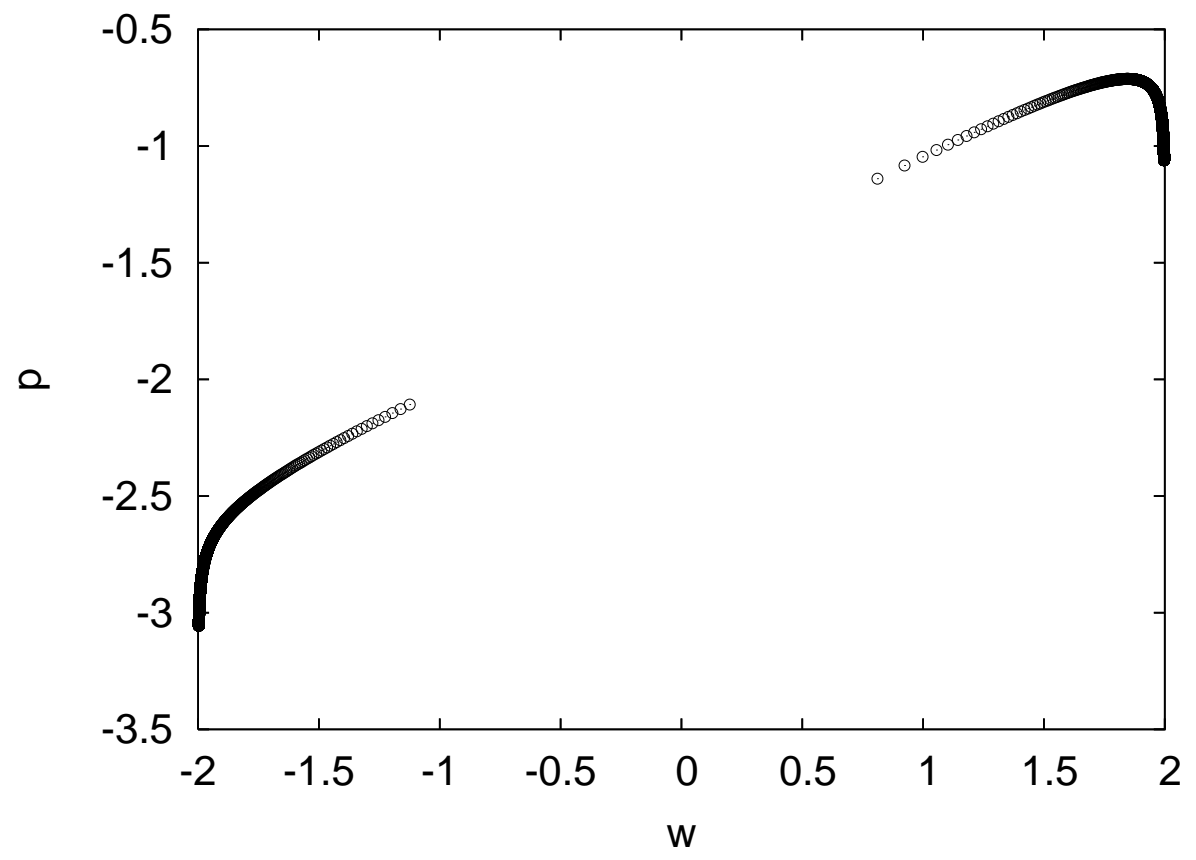




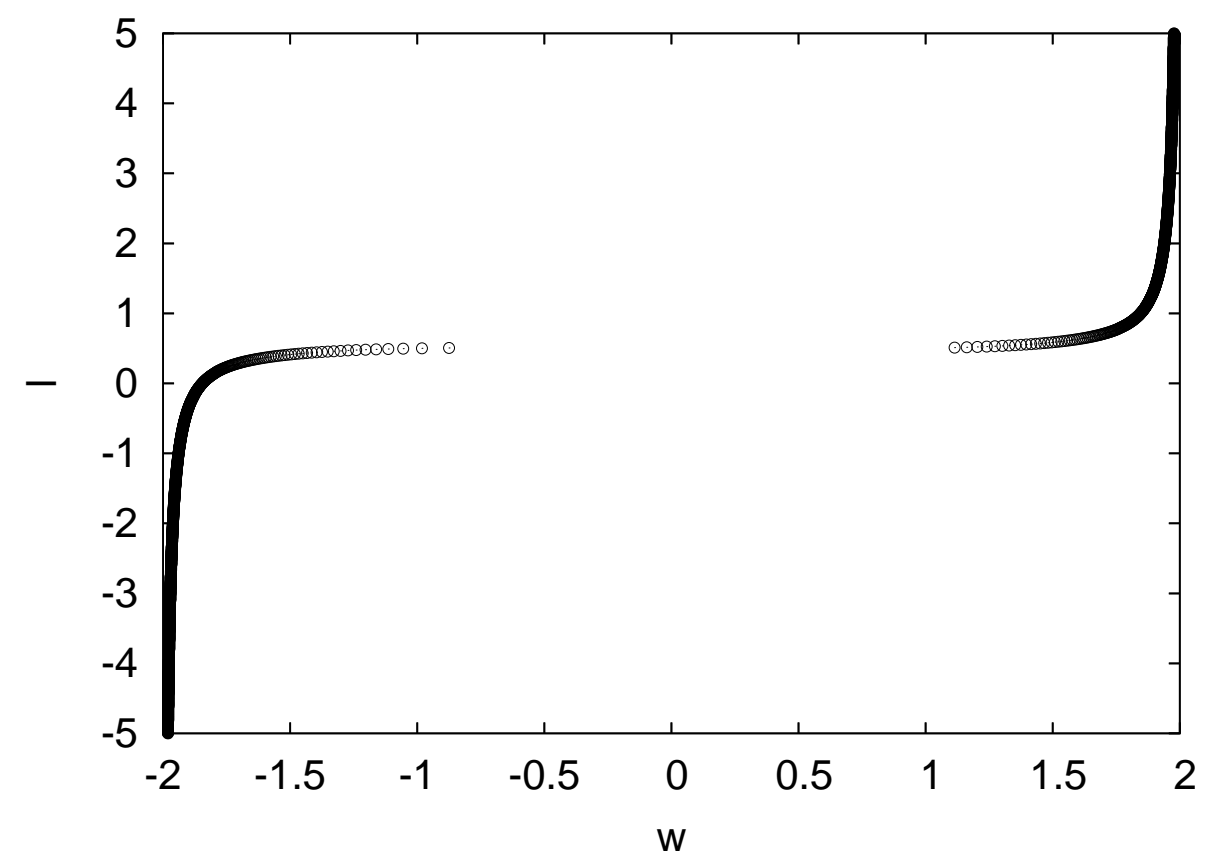




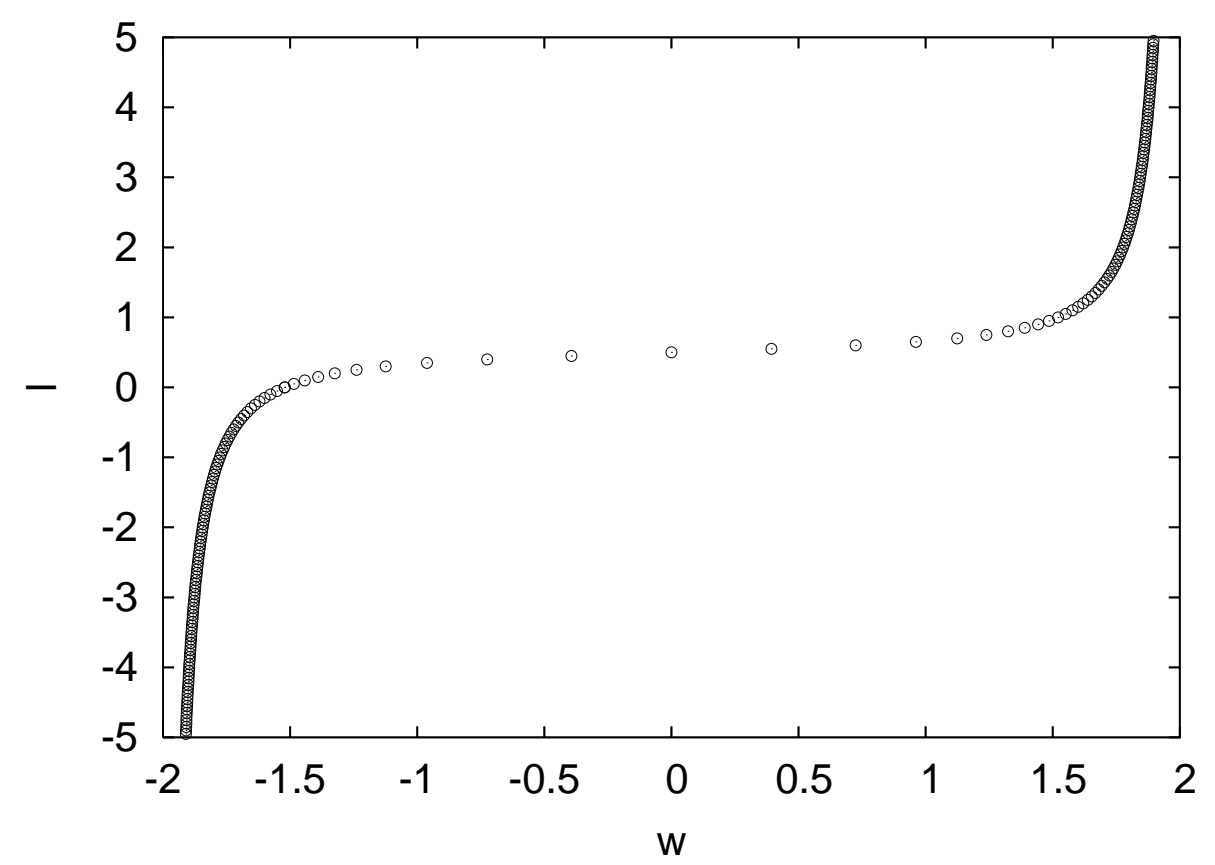

